\title{
T hema \\ Conditions à la transformation des pratiques langagières d'enquête en histoire scolaire: une étude de cas en primaire
}

\author{
Sylvain Doussot, Université de Nantes
}

L'article revient sur des études de cas qui montrent les capacités des élèves à mobiliser des pratiques qui ont à voir avec celles des scientifiques. Deux types d'enquêtes sont ainsi possibles en classe d'histoire : suivre des procédures de critique documentaire pour établir une vérité, ou articuler la recherche de la vérité par analyse des traces du passé et recherche du sens. Nous documentons les conditions du passage de l'un à l'autre de ces types d'enquête par l'analyse d'un cas de travail autonome d'élèves de fin de primaire. Ce cas questionne l'idée que le développement des compétences se ferait par la maitrise de règles décrivant la pensée historienne, et il met en lumière que des compétences scolaires peuvent aider les élèves à dépasser les habitudes de la discipline scolaire.

L'étude ${ }^{1}$ dont rend compte le présent article s'inscrit dans l'hypothèse de l'existence de communautés discursives scolaires propres à chaque discipline enseignée (Jaubert et al., 2003 ; Jaubert, 2007). Concernant l'histoire scolaire, les caractéristiques d'une telle communauté ont été largement étudiées par les recherches didactiques qui se sont intéressées aux finalités, contenus et pratiques de la discipline scolaire ${ }^{2}$ (histoire et/ou histoiregéographie). Les résultats de ces recherches nous fournissent des éléments pour décrire le fonctionnement dominant de cette communauté discursive historienne scolaire (CDHS), et donnent ainsi à voir par comparaison les cas dans lesquels le fonctionnement d'une classe s'en éloigne. Ce sont des cas de ce type que nous questionnons ici dans le but d'explorer les transformations du fonctionnement des classes d'histoire sous l'angle d'une modification des pratiques langagières ordinaires des élèves lorsqu'elles ou ils enquêtent sur le passé à l'aide de documents (Doussot, 2018a). Dans la perspective adoptée, le langage est pris à la fois comme objet d'observation, dans ses variations révélatrices de transformations, et comme facteur explicatif pour penser l'apprentissage disciplinaire et en particulier l'apprentissage de manières historiennes d'enquêter sur des traces du passé : selon l'hypothèse communauté discursive, apprendre une discipline c'est notamment transformer ses manières d'agir-parler-penser.

Deux points de vue peuvent être mis en œuvre pour appréhender ce type de transformation. L'un s'intéresse à la manière dont les élèves s'insèrent dans la CDHS, c'est-à-dire accèdent aux savoirs et pratiques de savoirs de la discipline scolaire et deviennent scolairement performant.e.s. L'autre, que nous privilégions, s'intéresse plutôt au passage de savoirs et pratiques de savoirs historiques scolaires, donc préconstruits, à des savoirs et pratiques de savoirs historiques scientifiques-scolaires, c'est-à-dire émergents. Par cette approche, nous visons à documenter les conditions de possibilité pour des élèves déjà inscrit.e.s dans une CDHS de passer à des pratiques (notamment) langagières ayant à voir avec celles des historiennes et historiens, autrement dit à une communauté discursive historienne scientifique-scolaire (CDHSS).

Pour parvenir à produire des savoirs didactiques sur les conditions de cette transformation, il est nécessaire de définir la norme épistémologique qui permet l'identification de ce passage. C'est ce qu'offre le cadre théorique de l'apprentissage par problématisation (Fabre, 2009; Orange, 2012) qui modélise sous un processus de construction - plutôt que de résolution - de problèmes les savoirs et activités propres à la démarche scientifique. La problématisation des historiennes et historiens constitue alors un modèle à partir duquel peuvent être analysées les situations singulières observées empiriquement (Doussot, 2017). Un tel modèle théorique, celui d'une histoire que l'on peut qualifier de critique et réflexive, peut servir de matrice à l'analyse de situations sortant de l'ordinaire de la CDHS afin d'en dégager les spécificités engageant une transformation en direction d'une CDHSS.

1 Cette étude prend appui sur les données produites à l'occasion d'un mémoire de master. Je remercie Lisa Faivre pour ce travail et l'accord pour son usage dans le présent article.

2 Voir notamment les travaux des années 1990 à l'INRP pour la France, pilotés par Audigier. Pour une approche plus récente et synthétique, voir par exemple Audigier et Tutiaux-Guillon (2008). 
Le présent article fait d'abord retour sur quelques études de cas qui montrent les capacités des élèves à fonctionner autrement dans la discipline scolaire histoire, en direction d'une problématisation scientifiquescolaire. Ce retour pointe la problématique centrale de l'étude : la transformation en jeu dans le passage d'une communauté discursive à l'autre ne relève pas de l'acquisition de procédures propres à une " pensée historienne " qu'il faudrait simplement appliquer, mais au développement des capacités des élèves à évaluer les situations d'étude afin de choisir quelles procédures sont pertinentes. Ce passage renvoie à deux types d'enquêtes différentes sur le passé : le premier s'identifie à des procédures de critique documentaire pour établir une vérité historique, le second, dont rend raison la modélisation en problématisation, pointe l'articulation entre la recherche de la vérité par analyse des traces du passé et la recherche du sens par production d'une explication cohérente et crédible 3 . Nous proposons ensuite de documenter les conditions du passage de l'un à l'autre de ces types d'enquête sur la base de l'analyse détaillée d'un cas observé dans un travail autonome d'élèves de fin de primaire. Parce qu'il «fait cas » (Passeron et Revel, 2005), ce corpus, d'une part, questionne les approches didactiques sur le développement des compétences des élèves en histoire qui le pensent sous l'hypothèse d'une maitrise de règles méthodologiques décrivant la pensée historienne ${ }^{4}$. Il ouvre d'autre part la discussion à des enjeux spécifiquement didactiques et heuristiques du point de vue de la pratique enseignante.

\section{Des capacités critiques des élèves peu sollicitées dans le fonctionnement de la discipline scolaire}

Le fonctionnement de la discipline scolaire histoire a depuis longtemps été décrit comme étant caractérisé par la faible intensité des activités du point de vue cognitif (Lautier, 1997), y compris lorsqu'elles consistent à faire étudier des documents. Deux opérations sont centrales dans ce fonctionnement ordinaire : la recherche d'informations ponctuelles dans des documents et la reproduction de connaissances préalablement apprises (TutiauxGuillon, 2008). En parallèle, plusieurs études récentes montrent que les élèves, même jeunes, sont en mesure de mobiliser d'autres capacités, y compris critiques et réflexives, lorsqu'il s'agit d'analyser des documents pour interpréter le passé.

C'est le cas de l'étude de Cariou (2016) qui propose de comparer deux classes de deux niveaux différents (primaire, élèves de 9-10 ans; secondaire, élèves de 13-14 ans) travaillant sur un même document (une allégorie éditée en 1789 présentant une critique des rapports entre les trois ordres de la société d'Ancien régime). L'analyse didactique opérée dans le cadre de la Théorie de l'action conjointe en didactique (TACD) révèle que par moments et pour certain.e.s élèves, même dans ces situations ordinaires, il y a transformation des activités (notamment langagières) d'un paradigme propre à la discipline scolaire - le paradigme de l'information - en un paradigme "indiciaire ", ainsi qualifié par référence à l'histoire scientifique telle qu'elle peut être pratiquée par les historiennes et historiens, dans la lignée de l'école des Annales et de la microhistoire. Dans ces moments singuliers et exceptionnels, les élèves en question mènent une enquête qui ne se limite pas à mettre en relation des idées et des faits, mais tend à opérer le contrôle des unes par les autres.

Dans un autre cadre théorique - celui de la problématisation - et sous une autre méthodologie - celle des "situations forcées" (Orange, 2010) - une étude récente (Doussot et Fink, 2019) réalisée dans une classe d'élèves de primaire (8-9 ans) met en lumière leur capacité sous-jacente à entrer dans une démarche critique de témoignages sur la vie quotidienne des générations précédentes (notamment sur la question des relations filles/ garçons de leur âge) qui donne accès à une réflexivité sur leur propres idées initiales. La situation co-construite par la chercheuse, le chercheur et l'enseignante permet la sortie d'une simple activité dominée par une approche réaliste des sources (Audigier, 1995) : les élèves, s'ils et elles commencent par trouver dans les témoignages des éléments confortant leurs idées préconçues (notamment la séparation réelle filles/garçons à l'école apprise l'année précédente), sont généralement ouvert-e.s à les mettre en discussion dès qu'ils et elles sont amené.e.s par la situation à confronter certains témoignages contradictoires, et à poursuivre l'enquête en proposant d'autres explications possibles. Dans ce deuxième exemple, à nouveau, les pratiques langagières et les positions des

3 Dualité que l’on peut référer à un enjeu de plus grande généralité dans les sciences sociales et que Berthelot (1990) repère comme " deux tendances ": "l'une à généraliser sans soucis de vérification empirique, l'autre à établir des faits quelle que soit leur pertinence théorique " (p. 14).

4 Pour ne prendre qu'une référence parce qu'elle synthétise de manière très actualisée les recherches états-uniennes dans cette approche de la pensée historienne, voir De La Paz et Nokes (2020). 
élèves par rapport aux énoncés qui circulent dans la classe (témoignages, propositions d'élèves, de l'enseignante, etc.) tendent alors à se rapprocher d'une démarche critique structurellement analogue à celle des historiennes et historiens confrontant critiques externe et interne des sources et permettant d'évaluer les hypothèses émises initialement.

Ces deux études, parmi d'autres, rendent visible un premier constat intéressant du point de vue de la transformation des manières de faire habituelles des élèves. Ils et elles ont des capacités peu sollicitées dans les situations ordinaires de la discipline scolaire qui se rapprochent pourtant des visées de développement des compétences disciplinaires issues de la discipline de référence, notamment des compétences critiques incarnées par l'enquête indiciaire. Dès lors, on peut faire l'hypothèse que favoriser la transformation de la CDHS en CDHSS ne consiste pas essentiellement dans le développement de ces capacités critiques, mais plutôt dans le développement de la capacité des élèves à mobiliser à bon escient ces modalités disponibles mais inhabituelles. Il est possible d'inscrire cette hypothèse dans le cadre des distinctions faites par Rey (2014) entre procédures et compétences : la compétence au sens fort du terme consiste à savoir interpréter, voire construire, les situations afin de mobiliser les procédures maitrisées « à bon escient ».

\section{De l'enquête d'histoire scolaire à l'enquête historienne critique et réflexive}

En quoi consiste cette compétence disciplinaire qu'il s'agirait de viser dans l'enseignement d'une histoire analogue à celle des historiennes et historiens? Pour répondre à cette question, il est nécessaire de référer à une norme épistémologique susceptible de rendre compte des principes de la discipline scientifique valables dans sa version scolaire. Le modèle élaboré de problématisation historique ne consiste donc pas à imaginer que les élèves peuvent enquêter comme les scientifiques, mais il ne consiste pas davantage à abandonner l'idée que l'histoire scolaire doit avoir quelque chose à voir avec l'histoire scientifique. Ce quelque chose réside dans ces principes épistémologiques comme modèle théorique de l'enquête.

Cette modélisation repose sur deux dimensions (Doussot, 2017). D’une part, elle porte sur deux objets : le passé, c'est-à-dire l'évènement ou le phénomène historique en jeu (comme les rapports filles/garçons dans les années 1950), et l'enquête sur le passé. Celle dernière implique la dimension réflexive de ce type d'enquête. En effet, il ne s'agit pas seulement de s'intéresser aux documents qui donnent accès au passé mais aussi à la manière dont on les utilise dans la classe. Dans une perspective Deweysienne, inspiratrice du cadre de la problématisation, il s'agit de considérer l'activité visée comme une enquête sur l'enquête elle-même (Fabre, 2017). Du point de vue des historiennes et historiens, comme le montre l'étude de Grafton (1998), cela signifie qu'il s'agit de " dire l'histoire de leurs recherches aussi bien que l'histoire des objets dont ils [et elles] trait[ai] ent, à deux niveaux séparés et dans des temps différents" (p. 174). De même, pour la recherche qui mène Ginzburg à son ouvrage "Le fromage et les vers ", " les obstacles qui se dressaient contre cette recherche appartenaient à la documentation, ils devaient donc faire partie du récit ; tout comme les hésitations et les silences du protagoniste face aux questions de ses persécuteurs - ou face aux miennes »(Ginzburg, 2010, p. 385). Sous ce premier principe, l'analyse didactique doit mettre au jour les pratiques de production de récit de l'enquête elle-même et en particulier celles qui permettent un retour sur les premières idées explicatives, c'est-à-dire qui mettent au travail les représentations envisagées alors comme les objets principaux d'analyse (plutôt que les documents). C'est en effet à cette condition que l'explication est scientifique : en décrivant l'enquête, l'explication scientifique rend raison de la solution choisie et ne se contente pas de l'énoncer; elle rend compte des solutions envisagées et écartées.

D'autre part, la modélisation implique le principe de la mise en relation systématique du travail sur ce que disent les sources et sur ce qu'elles sont, c'est-à-dire en contrôlant les discours qu'elles portent (interne) et les conditions de leur production (externes).

La finalité de cette combinaison est de traiter du problème en jeu comme étant constamment contraint non seulement par le sens, mais aussi par le réel des sources. Cette dimension critique doit conduire à observer dans le corpus les modes d'appréhension des documents par les élèves entre réalisme (pour lequel critiques externe et interne sont séparées, la source donnant un accès direct au passé) et critique (manifestant des pratiques langagières de corroboration entre les sources et de contrôle du sens de l'explication par les faits portés par les sources permettant la construction d'hypothèses de plus en plus productives).

La combinaison de ces deux principes, réflexif et critique, rend possible le contrôle autonome du cheminement de l'enquête parce qu'il permet de varier les procédures disponibles lorsque les hypothèses ne se vérifient pas, sans clore le processus (Rey, 2014). 
La modélisation proposée doit s'entendre dans notre étude non comme une référence servant à évaluer si une histoire critique et réflexive est atteinte, mais plutôt pour observer ce qui, dans les pratiques, notamment langagières, ordinaires de la discipline scolaire, rend possible cet aboutissement. L'enjeu étant l'identification des conditions de possibilité d'une transformation des pratiques d'enquête, il s'agit d'appliquer la comparaison entre le modèle et l'ensemble de la situation.

\section{Une situation qui fait cas pour l'étude didactique}

Le cadrage de la recherche par la modélisation sert à l'analyse de situations empiriques, mais celles-ci n'ont de pertinence dans un champ de recherche qu'à la condition de permettre le dépassement des connaissances déjà acquises. Pour y parvenir, il est indispensable de se confronter à un corpus qui met en discussion ces connaissances pour les amender ou les approfondir, voire les mettre en cause. La situation proposée 5 y répond par plusieurs caractéristiques.

En premier lieu, la classe et en particulier les deux élèves dont il est question dans ce corpus sont scolairement performants en histoire. Cela signifie qu'ils maitrisent les attentes de l'enseignante sur la plupart des points, qu'ils soient disciplinaires ou plus génériques. L'attention qu'ils montrent à la formulation des réponses aux questions sous forme de phrase (voir en $27^{6}$ : "non on fait une phrase ! ) illustre cette dimension générique, tout comme le respect scrupuleux des consignes de couleur (question 4 et tours de parole 35-51). Nous détaillons ci-après les données manifestant la maitrise disciplinaire scolaire de ces deux élèves. Ce premier point fait du corpus un cas parmi les études empiriques de ce type en didactique de l'histoire : leur travail collectif autonome donne un accès rare à ce que cette maitrise permet, aux usages qu'ils font en acte de leurs ressources scolaires.

En second lieu, les données tirées de la situation d'étude de documents de ces deux élèves ne montrent pas seulement un respect des attentes et une performance scolaire : elles montrent qu'ils sortent régulièrement de l'enquête d'histoire scolaire et du mode réaliste d'appréhension des documents. En ce sens, ce corpus fait cas par rapport aux études des deux types dominants dans le champ : soit des situations ordinaires montrant des activités scolaires plus ou moins conformes aux attentes, soit des situations " forcées " dans lesquelles des interventions co-construites cadrent la sortie de la forme scolaire disciplinaire dominante. En l'espèce, les élèves sortent de leur propre mouvement des attentes pour aller vers une enquête et des pratiques ayant à voir avec les principes de l'enquête scientifique. Autrement dit, ce corpus donne accès à des conditions " naturelles " de transformation des pratiques (nous verrons notamment que l'enseignante ne favorise pas cette transformation).

Nous proposons de faire une première description du corpus pour donner corps à cette pertinence du cas. Il s'agit de donner à voir dans un premier temps les différentes composantes de l'activité langagière déployée par les deux élèves, selon les critères définis précédemment, ainsi que les manières dont elles s'articulent dans un processus de recherche, qu'il s'agit de caractériser. Cette description prend explicitement appui sur les documents en annexe.

La lecture de la transcription des échanges montre un processus continu de recherche de la part de ces deux élèves, en décalage avec les pratiques ordinaires qui réduisent ce processus à un jeu de questions/réponses sans travail de vérification. On peut qualifier ce cheminement de processus de recherche dans le sens d'une enquête au moins partiellement problématisante dans la mesure où il n'est pas limité à la production d'une réponse, vraie au regard d'éléments documentaires, ou ayant du sens sans souci de vérité. Ce constat est particulièrement visible concernant la première question qui est traitée au début (1-11) puis reprise (57-115) : ils s'abstiennent de noter la première idée qui leur vient (8-9), ils relisent plusieurs fois les documents pour vérifier leur réponse, et ils s'abstiennent de demander la solution à l'enseignante (on l'observe précisément en 79 : Théo ne demande pas la réponse à la question mais explique qu'ils ne comprennent pas, et esquisse en 81 l'explication de ce qu'ils ne comprennent pas). Ce processus est remarquable parce qu'il manifeste une sortie des pratiques dominantes

5 La situation est issue d'un mémoire de master en didactique (Faivre, 2018) qui fournit les éléments du corpus utilisé ici. Le corpus est composé de la fiche " étude de documents " qui a été distribuée aux élèves (annexe 1), de la transcription des échanges d'un groupe de deux élèves au travail pour répondre aux questions posées dans cette étude (annexe 2) et de la fiche de préparation de l'enseignante ; les élèves disposent de 20 minutes pour ce travail et peuvent poser des questions et ainsi se faire aider par l'enseignante. La situation dans laquelle se trouvent ces élèves est classique pour cette classe de double niveau (élèves de 9 à 11 ans) dont la majorité des élèves provient d'un milieu social favorisé et a un niveau scolaire au moins satisfaisant. Ils et elles doivent lire et observer des documents pour répondre à des questions, ce qu'ils et elles font aussi dans d'autres disciplines (sciences, français notamment).

6 Les nombres correspondent aux tours de parole de la transcription en annexe 2. 
de la discipline scolaire qui consiste à viser avant tout à répondre aux questions, parfois à tout prix (au prix du sens ou des contraintes empiriques portées par les documents) et souvent en abandonnant certaines questions.

Ce processus de recherche constant n'est cependant ni uniforme ni analogue à un processus de problématisation abouti. Deux illustrations de l'absence d'enquête critique, entendue comme mise en tension d'idées et de faits relevant tant du contenu que de la nature des documents, sont intéressantes à pointer. La première concerne la $4^{\mathrm{e}}$ question (frise chronologique). Les deux élèves ne se préoccupent pas du tout des ambigüités du document (critique interne) concernant le premier vol en avion (le court texte évoque Clément Ader qui " fait décoller " son avion sur 50 mètres en 1890 et « le premier vol en 1903 » des frères Wright, et les élèves s'arrêtent sans discussion ni contrôle à la première date). La seconde illustration de l'absence d'enquête critique concerne le $3^{\text {e document }}$ de la première question : les élèves ne s'intéressent pas à la date de ce document (1856). En ignorant ainsi la critique externe, ils ne peuvent mettre en discussion le contenu factuel du texte autrement qu'en relation avec leur idées explicative (8-9) de ce qui est écrit, et en particulier avec les temporalités multiples en jeu (nous y revenons plus loin : le texte commence ainsi « depuis douze ans, des changements notables..." mais les élèves semblent le traiter comme un document d'aujourd'hui : voir l'usage du présent (5) : « on peut acheter aux fermiers tout le lait disponible...»). Par ailleurs, leur enquête principale, sur la première question, n'aboutit pas complètement comme problématisation critique et réflexive sur le passé. En effet, ils concluent (114 et 115) que le chemin de fer a pour conséquence de "transporter le lait à plus grande distance ", ce qui n'est pas faux, mais ne rend pas compte de l'enquête qu'ils ont menée et notamment de la critique (réflexive) de leur première compréhension ; le récit de l'évènement n'est pas accompagné de celui de l'enquête, qui permettrait de rendre raison de la réponse.

Pourtant l'activité déployée par ces élèves relève parfois des deux principes de l'enquête historienne. En premier lieu, les élèves sont systématiquement dans la visée de comprendre et pas simplement de donner une réponse (3: «je comprends pas trop la question »; $7:$ "j’ai pas pigé »; $23:$ " tu comprends la question ?»; 72 : parce que je comprends pas bien »; 79: «celle-là on comprend pas »; 81 : « mais avec le lait on comprend pas "; 85 et 87 : «j'ai pigé »). Ce qui se confirme par le tournant que constitue justement le moment de compréhension (85 et 87) : dès lors ils sont d'accord pour passer à la rédaction de l'explication (93 : «on sait ça veut dire quoi mais on sait pas comment expliquer dans notre phrase »). La réticence à donner une réponse sans compréhension figure la construction du problème par le besoin de formuler des raisons de répondre de telle ou telle manière. Mais l'engagement vers une enquête historienne est plus directement visible dans le travail qu'ils effectuent ensemble sans l'aide de l'enseignante, notamment par des relectures systématiques des documents (en 61 quand les élèves s'attaquent à nouveau à cette première question). Ce qui conduit Adrien à travailler à nouveau le texte, mais armé du travail précédent et donc d'une meilleure compréhension de la question. En 66 , il identifie finalement la pertinence de la première phrase du texte (" attends... ten... ten... il y a écrit "depuis 10 ans [sic] des changements notables se sont produits dans le mode d'approvisionnement du lait"... ») : ce constat de changement dans le passé (et non entre le passé et le présent) est une information du texte qui devient un indice pour lui parce qu'il sait mieux à ce stade ce qu'il cherche. De même, ils tentent de corroborer leur hypothèse (difficile à qualifier cependant à partir du corpus) sur un document par la lecture des autres documents (en 75, Théo confronte leur incompréhension du texte - troisième document - avec l'explication donnée par le deuxième document). Ce qui vaut ici, didactiquement, c'est l'usage à bon escient d'une règle de la pensée historienne qu'est la corroboration7. Cet usage est peu ordinaire et repose sur la dynamique de "re-cherche ", c'est-à-dire de volonté de contrôle de l'intérêt d'une information par croisement avec une autre, par l'activité de " re-lire » un document armé d'une hypothèse. Cependant, malgré ces démarches propres à une enquête problématisante en histoire, l'absence de certaines connaissances (par exemple la différence entre approvisionnement et fabrication - 67-68) et de ressources pour y palier conduit rapidement à la demande d'aide auprès de l'enseignante.

\section{Des compétences scolaires points d'appui pour dépasser la discipline scolaire}

L'étude du corpus par les catégories de la discipline scolaire et celle du modèle théorique de l'enquête comme problématisation fait également ressortir la continuité du processus de recherche observé avec certaines compo-

7 Un des euristics identifiés par Wineburg (1991) pour décrire par des règles la pensée historienne. 
santes traditionnelles de la discipline scolaire, ce qui laisse entrevoir les manières dont les élèves prennent appui sur celles-ci pour les dépasser.

Les deux élèves s'accordent sur le fait que comprendre en histoire, c'est trouver une cause. On le discerne dans leur volonté de mettre en rapport « lait » et " chemin de fer » dès le début de leurs échanges $(5$ et $7:$ : du lait pour faire des chemins de fer ?"; 24 : «donc c'est peut-être grâce aux marchandises le développement des chemins de fer et de la navigation »; 71 : « ... c'est grâce au lait »). La forme causale de la réponse attendue les incite à construire et donc à rechercher une relation causale satisfaisante, ayant du sens. Celle entre le lait et le chemin de fer qu'ils envisagent n'en ayant pas, ils poursuivent leur investigation. D'une certaine manière la contrainte de la causalité attendue leur sert de boussole (Fabre, 2011) dans cette situation d'incertitude dans laquelle l'hypothèse issue du document est contredite par l'absence de sens de l'explication qui en découle. Il leur faut changer de manière de faire et en particulier interroger l'enseignante.

Un deuxième point d'appui est constitué par la compréhension qu'ils ont de l'attente de l'enseignante de réponses précises aux questions posées et non de manière approximative (par exemple un contrôle est fait par Adrien du respect de la consigne par Théo pour remplir, selon les bonnes couleurs, la frise chronologique (35); ou en 62 et en 93, nouvelle lecture de la question 1 pour la confronter à une nouvelle lecture du texte). Cette manière de procéder est liée à deux habitudes de la discipline scolaire que maitrisent ces deux élèves scolairement performants. D'une part à la recherche d'informations : ils savent qu'il faut rapprocher la question et les documents, trouver dans ces derniers des éléments qui font directement écho aux questions posées (par exemple en 24 : « en fait là il y a écrit [...] et là il y a écrit [...]. Donc c'est peut-être grâce aux marchandises le développement des chemins de fer »). D’autre part, l'attention aux questions est liée aux retours récurrents des élèves aux documents : ils relisent dès qu'ils sont bloqués $(3:$ "attend, je relis le texte "; 7 : « attend on relit »; 23 : Adrien relit ; 61 : " tu relis dans ta tête »). Les relectures participent directement à la mise en question et au travail d'une première compréhension dans ce mouvement réflexif propre à la problématisation. On peut également associer ces retours réflexifs aux habitudes d'écriture. Les attentes de l'enseignante et de l'école plus généralement sont traduites sur ce plan de manière très contraignante par ces deux élèves. Ainsi, Théo met-il en garde son camarade (27: " non on fait une phrase! ») contre le fait de ne rendre compte que d'une information (26 : " du coup on écrit la marchandise »), ce qui les pousse à revenir sur leur compréhension pour bien la formuler sous forme d'explication. C'est également cette exigence d'écriture qu'ils s'imposent qui les conduit à revenir vers l'enseignante (99: "On n'arrive pas à le mettre sur la phrase ", alors que Théo avait " pigé »). Compréhension et explication sont ici dissociées en acte par ces deux élèves, jusqu'à la formulation finale (107 : "ça permet de transporter ") confirmée par l'enseignante (108) et inscrite (115 et 116). Cependant, nous l'avons souligné, l'enquête ne va pas jusqu'à un texte rendant compte de raisons - qui caractérisent un savoir problématisé - et Théo lui-même apparait insatisfait, malgré sa formulation validée : il répète, même après validation, "Mais on n'arrive pas à former notre phrase..." (111).

Il nous semble à ce stade de l'analyse que pour donner du sens à cet échec relatif et ainsi mieux caractériser les potentialités offertes par l'étude de ce cas pour comprendre les conditions d'une transformation des pratiques langagières d'enquête, il est nécessaire de prendre un point de vue plus radicalement didactique et revenir aux contraintes proprement disciplinaires, au-delà de la généricités des concepts épistémologiques (hypothèses, causes, raisons...) ; il faut notamment se demander de quelles raisons il s'agit ici, en histoire et à propos de cet évènement du milieu du $19 \mathrm{e}$ siècle.

\section{L'importance d'un problème proprement disciplinaire}

Pour expliquer l'insatisfaction des élèves et l'enquête historienne finalement avortée, on pourrait se contenter de faire jouer les catégories génériques d'analyse épistémologique. On pourrait souligner que des concepts ne sont pas connus des élèves et que cette ignorance les empêche d'avancer : on l'a constaté concernant la différence entre fabrication et approvisionnement (66-68). L'enquête des élèves devrait ici être dirigée vers ce processus de conceptualisation techno-économique. Une telle analyse est en l'occurrence fondée, car une des spécificités de l'histoire est d'être une « discipline du contexte " social et non une " science sociale particulière " ou "spécialisée " (Passeron, 2006, p. 92). De ce fait les questions qu'elle traite peuvent toucher tous les domaines du monde social, ce qui contraint à des connaissances parfois pointues, comme ici en termes de production industrielle. Mais, si l'on restait à des explications de niveau générique, on pourrait également penser que les deux élèves ne maitrisent pas le couple conceptuel cause/conséquence puisqu'ils imaginent que le lait $(5-7 ; 71 ; 75)$, ou même les marchandises seraient les causes du développement des transports (24: " c'est peut-être grâce aux marchan- 
dises le développement des chemins de fer »). Or c'est plutôt une méconnaissance factuelle - sans doute sur le fonctionnement de la machine à vapeur - qui les bloque et qui relève, on vient de le voir, de la spécificité de la discipline générale du monde social qu'est l'histoire (scientifique comme scolaire). C'est même un des obstacles principaux que rencontrent les enseignantes et les enseignants de cette discipline à concevoir des situations d'étude documentaire, puisque chaque évènement ou phénomène étudié relève du singulier du monde social et peut nécessiter des connaissances spécialisées qui ne sont pas " au programme "; cette contrainte disciplinaire fait de chaque étude non pas un exercice relevant d'une compétence technique à laquelle on pourrait entrainer les élèves, mais plutôt un cas particulier. C'est cette contrainte qui réclame une compétence d'enquête exigeante, bien au-delà de l'application de procédures.

L'importance des déterminants proprement disciplinaires se révèle également dans la centralité du problème de temporalités qui revient à plusieurs reprises dans notre analyse. L'absence d'attention des élèves envers la date (1856) du document 3 de la première question, l'usage du présent dans leur compréhension du même texte, la manière dont Adrien est intrigué par la relecture du début du texte, qui devient un indice (66 : « Attends [...] Il y a écrit depuis 10 ans des changements notables [...]») en situant le changement dans le passé, et surtout les interventions décisives de l'enseignante (82, 100 à 110) mettent au jour le rôle joué par la difficulté propre à l'histoire d'articuler les rapports passé/présent autrement que dans un sens commun linéaire et chrono-causal. On peut faire l'hypothèse - que nous explorons pour terminer - que c'est cette compétence fondamentale de la discipline historique - articuler plusieurs temporalités et régimes de causes - qui pose problème à ces deux élèves performants et les empêche d'aller au bout de leur enquête.

La transcription des échanges met en lumière deux temps de compréhension des élèves et la persistance de leur difficulté à expliquer. L'exclamation d'Adrien (83: "Ah ! le lait il va à Paris avec les trains ? ") manifeste sa mise en sens par élimination de l'hypothèse du lait faisant fonctionner les trains : il y a un sens bien plus plausible au document 3, le lait est transporté par le train. Pourtant, cela ne leur suffit pas pour répondre à la question qui leur est posée et qu'il faut rappeler : " quelles sont les conséquences du développement des chemins de fer sur la commercialisation du lait dans la capitale ? ». Ils donnent désormais un sens acceptable au document, mais ce sens ne répond pas directement à la question posée : il n'est pas pertinent. On atteint ici le point limite qui laisse les deux élèves au seuil des principes d'une enquête scientifique en histoire. Le sens du document est désormais empiriquement vérifié, mais il ne permet pas de produire une explication du processus historique en jeu dans la question posée. Du point de vue de la problématisation, on peut dire qu'il leur manque un savoir de la question. Le processus d'enquête peut en effet être décrit comme le rapprochement à tâtons de la question, des hypothèses et des faits (présents dans les documents et dans la tête des élèves). Pourquoi ne comprennent-ils pas la question?

L'explication didactique à ce blocage est à rechercher dans l'épistémologie de l'histoire dont un des apports essentiels est de spécifier les types de problèmes en jeu dans la discipline. Les élèves ne comprennent pas la question parce qu'ils en restent à leur connaissance actuelle du monde des transports et ne peuvent pas voir le changement en question puisque pour eux le fait de transporter des marchandises (en train notamment) est une évidence d'expérience : si le passé est identique au présent, il leur est impossible de penser un changement. Leur problème historique n'est donc pas la question de l'enseignante (comprendre les effets de l'invention du $\operatorname{train}^{9}$ ) : le difficile pour eux consiste à voir qu'avant l'invention du train les possibilités de transport étaient réduites. C'est l'écart entre ce que savent les élèves et le savoir visé (ici sur le changement) qui détermine l'enjeu de savoir possible de la situation didactique : ils savent ce que peut faire un train mais ne savent pas comment on s'approvisionnait avant son invention.

Cette explication épistémologique est par ailleurs confortée par les explications fournies par l'enseignante aux élèves. Elle souligne à de multiples reprises dans sa paraphrase du document l'écart entre avant et maintenant (voir en 100, 104, 106, 108 et 110). Cependant, elle le fait de manière peu systématique et peu hiérarchisée, sans être guidée par une analyse préalable du problème historique des trois temporalités à articuler (avant 1850, après 1850, aujourd'hui) et notamment la première. Ce qui ne permet pas d'éclairer complètement les élèves pour qu'ils soient eux-mêmes en mesure de comprendre la question puis de la rapprocher de leur compréhension du texte. Les élèves restent dans l'expectative (111: " mais on n'arrive pas à former notre phrase ») et se contentent d'une explication non justifiée par l'enquête.

8 Voir une étude de ce phénomène didactique dans Doussot (2018b).

9 Dans sa fiche de préparation, elle formule ainsi son objectif de séance : " comprendre le rôle du développement des transports ». 


\section{Conclusion}

Larticulation des temporalités multiples est un des problèmes types fondamentaux en histoire. Ces problèmes scientifiques sont, par nature, explicatifs, ils mettent donc en jeu le rapport entre la compréhension spontanée que les historiennes et historiens ou les élèves peuvent avoir des situations passées et la nécessité de mettre en texte les ressorts de cette explication et d'autres possibles. Aussi peut-on appréhender ce qui se passe pour ces élèves, et - puisque le corpus qui en rend compte fait cas dans le champ de la didactique de l'histoire - des conditions de possibilité d'une enquête historienne scientifique-scolaire, en articulant compréhension et explication sous l'angle d'une problématique disciplinaire. La compréhension des documents ne suffit pas puisqu'elle est possiblement indépendante de la compréhension de la question posée. C'est dans le processus d'explication historienne, critique et réflexive, que ces deux compréhensions peuvent être confrontées, car il rend compte de l'activité compréhensive, qui se fait spontanément, en prenant pour objet les images et les idées préconçues des élèves. C'est ainsi bien d'une enquête sur l'enquête dont il s'agit, spécifiée ici par la discipline puisque cette activité compréhensive porte notamment sur les traces du passé et sur le rapport au présent des élèves.

Notre analyse didactique du cas s'est révélée nécessaire pour rendre raison de ce qui avance et ce qui bloque l'accès des deux élèves à un langage disciplinaire scientifique-scolaire qui unifie savoirs et pratiques de savoirs. Elle donne ainsi à voir des conditions d'une telle transformation, et par là de l'action transformative des enseignantes et des enseignants, dans le cas d'une enquête dont l'enjeu est l'articulation de temporalités multiples.

\section{Bibliographie}

Audigier, F. (1995). Des savoirs scolaires en question entre les définitions officielles et les constructions des élèves. Spirale, 15, 61-89.

Audigier, F. et Tutiaux-Guillon, N. (2008). Compétences et contenus : Les curriculums en questions. De Boeck Supérieur.

Berthelot, J.-M. (1990). L’intelligence du social. Le pluralisme explicatif en sociologie. Presses universitaires de France.

Cariou, D. (2016). Information ou indice ? Deux lectures d'une image en classe d'histoire. Revue française de pédagogie. Recherches en éducation, 197, 63-78.

De La Paz, S. et Nokes, J. (2020). Strategic Processing in History and Historical Strategy instruction. Dans D. L. Dinsmore, L. K. Fryer et M. M. Parkinson (dir.), Handbook of Strategies and Strategic Processing. Taylor and Francis Group.

Doussot, S. (2017). Modélisation des problématisations historiques en classe et chez les historiens. Recherches en éducation, 29, $22-37$.

Doussot, S. (2018a). L'apprentissage de l'histoire par problématisation. Enquêter sur des cas exemplaires pour développer des savoirs et compétences critiques. Peter Lang.

Doussot, S. (2018b). Lincoln et l'esclavage : Étude de cas de problématisation en histoire scolaire. Didactica Historica, 4, 99-105.

Doussot, S. et Fink, N. (2019). Faire problématiser des élèves de CE2 en histoire à partir de témoignages. Recherches en Didactiques. Les Cahiers Théodile, 27, 91-103.

Fabre, M. (2009). Philosophie et pédagogie du problème. Vrin.

Fabre, M. (2011). Eduquer pour un monde problématique. La carte et la boussole. PUF.

Fabre, M. (2017). Qu'est-ce que problématiser? Vrin.

Faivre, L. (2018). Etude documentaire en histoire au cycle 3 : Enjeux disciplinaires et littérature de jeunesse [Mémoire de master, université de Nantes].

Ginzburg, C. (2010). Le fil et les traces : Vrai faux fictif (Traduit par M. Rueff). Verdier.

Grafton, A. (1998). Les origines tragiques de l'érudition: Une histoire de la note en bas de page. Seuil.

Jaubert, M. (2007). Langage et construction de connaissances à l'école : Un exemple en sciences. Presses Universitaires de Bordeaux.

Jaubert, M., Rebière, M. et Bernié, J.-P. (2003). L'hypothèse " communauté discursive ": D’où vient-elle? Où va-t-elle? Les cahiers THEODILE, 4, 51-80.

Lautier, N. (1997). À la rencontre de l'histoire. Presses universitaires du Septentrion.

Orange, C. (2010). Situations forcées, recherches didactiques et développement du métier enseignant. Recherches en éducation, $H S(2), 73-85$

Orange, C. (2012). Enseigner les sciences : Problèmes, débats et savoirs scientifiques en classe. De Boeck.

Passeron, J.-C. (2006). Le raisonnement sociologique: Un espace non poppérien de l'argumentation. Albin Michel.

Passeron, J.-C. et Revel, J. (2005). Penser par cas. EHESS. http://cat.inist.fr/?aModele=afficheN\&cpsidt=16790137

Rey, B. (2014). La notion de compétence en éducation et formation. De Boeck.

Tutiaux-Guillon, N. (2008). Interpréter la stabilité d'une discipline scolaire : L'histoire-géographie dans le secondaire français. Dans F. Audigier et N. Tutiaux-Guillon (dir.), Compétences et contenus (p. 117-146). De Boeck Supérieur.

Wineburg, S. S. (1991). Historical problem solving : A study of the cognitive processes used in the evaluation of documentary and pictorial evidence. Journal of Educational Psychology, 83(1), 73-87. https://doi.org/10.1037/0022-0663.83.1.73 
T h e m a

\section{Annexe 1}

Étude de documents

Nom :

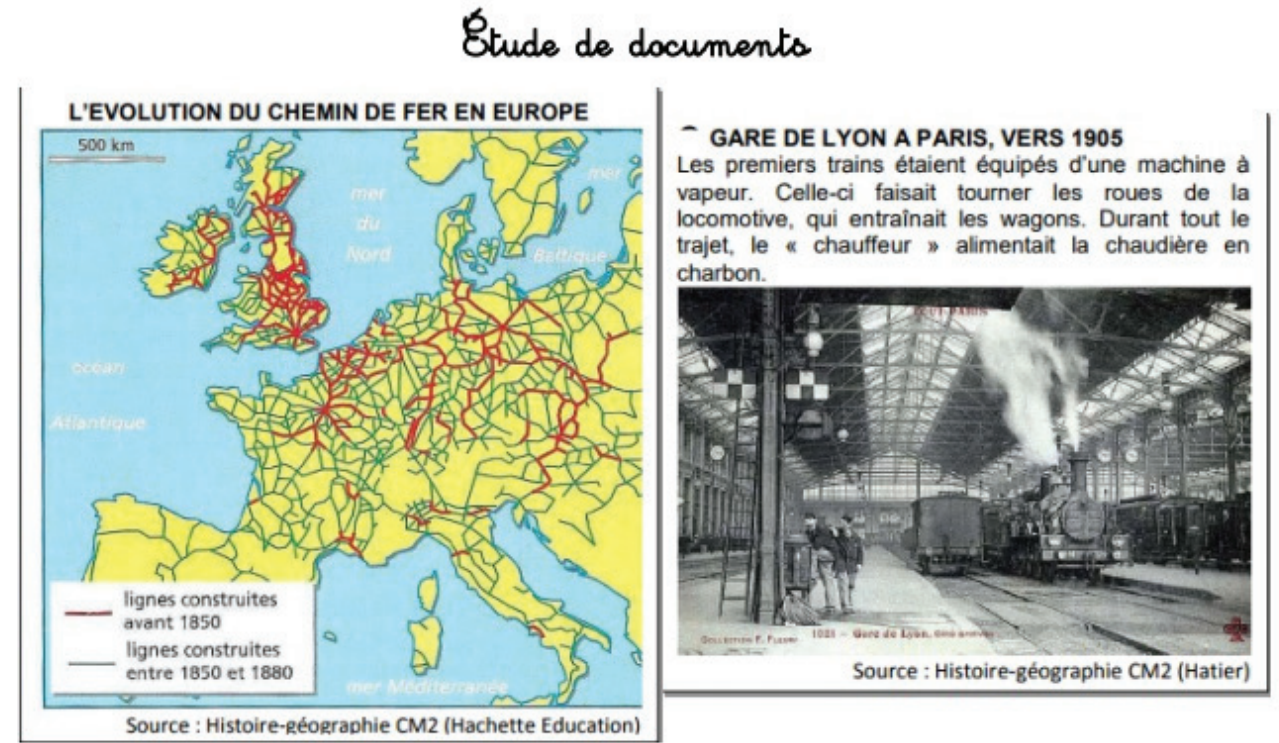

\section{L'approvisionnement en lait de Paris}

Depuis douze ans, des changements notables se sont produits dans le mode d'approvisionnement du lait; la mise en activité des grandes lignes de chemins de fer a procuré des moyens de transport puissants et économiques. Des entrepreneurs ont pu acheter aux fermiers tout le lait disponible provenant de leurs étables et l'amener à Paris. Ce nouvel état de choses, en étendant le cercle de l'approvisionnement et en augmentant considérablement les arrivages, a eu pour effet d'abaisser, dans une forte proportion, le prix de cet objet de consommation.

D'après Armand Husson, Les Consommations de Paris, 1856.

1 - Quelles sont les conséquences du développement des chemins de fer sur la commercialisation du lait dans la capitale? 


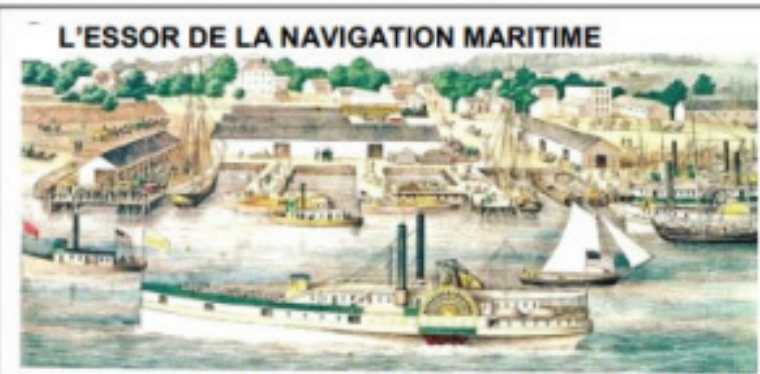

2 - Qu'est-ce-qui a

permis

A partir de 1850 , les bateaux à vapeur appelés «steamers» sont construits en tole. Ils peuvent transporter beaucoup plus de marchandises et de passagers que les bateaux à voiles. Le commerce maritime connalt ainsi un grand essor. Pour accélérer les traversées, des canaux transocéaniques sont percés : le canal de Suez (1869) entre la Méditerranée et la Mer Rouge, le canal de Panama (1914) entre l'Atlantique et l'Océan Pacifique. Source : histoire-géographie $\mathrm{cm} 2$ (hachette éducation)

3 - Qu'a permis le développement des chemins de fer et de la navigation maritime? d'accélérer les traversées ?

\section{UNE MOBILITE ACCRUE}

Le chemin de fer et la navigation maritime multiplient les possibilités de déplacement des idées, des hommes et des marchandises. Les matières premières et les produits industriels sont transportés sur de grandes distances. Des régions autrefois isolées s'ouvrent au monde moderne.

Source : Histoire-géographie CM2 (Hachette Éducation)

\section{LA NAISSANCE DE L'AVION}

L'aviation est à ses débuts : en 1890, Clément Ader fait décoller sur 50 mètres environ un premier engin et les frères Wright réalisent le premier vol en 1903.

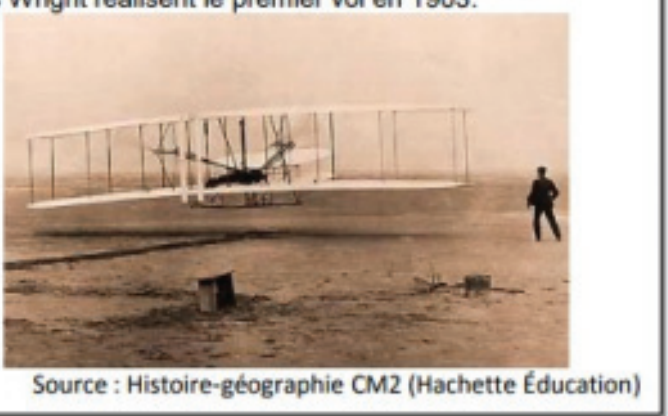

LA NAISSANCE dE L'AUtomobILE Et DE L'AVION

L'automobile à essence nait en 1866. A ses débuts, elle coûte très cher et les fabricants n'en produisent parfois que quelques exemplaires. En 1914, seules les usines de Paméricain Henry Ford fabriquent des automobiles en série.

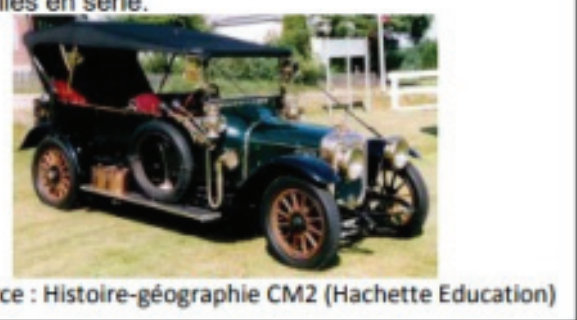

4 - Entoure en rouge sur la frise la date de naissance de l'avion, et en bleu la date de naissance de l'automobile. 
Annexe 2

Transcription des échanges

1. Théo : Alors on répond à la première question?

2. Adrien : Moi j’ai pas terminé.

3. Théo : Quelles sont les conséquences du développement des chemins de fer sur la commercialisation du lait dans la capitale ? Je comprends pas trop la question... Attends je relis le texte !

4. Adrien : Ah j’ai trouvé ! Commence à lire dès là [montre le texte].

5. Théo : Ah ouais... Du coup ça fait des entrepreneurs, on peut acheter aux fermiers tout le lait disponible provenant de leur étable et l'amener à Paris... Mais pour faire des chemins de fer ? Du lait ?

6. Adrien : Hein?

7. Théo : Du lait pour faire des chemins de fer ? J'ai pas pigé ! Attends on relit. C'est du lait pour faire les chemins de fer, j’ai pas pigé mais...

8. Adrien : C'est peut-être...

9. Théo : Je crois qu'on va passer plutôt là... [montre le verso de la feuille]

10. Adrien : Ah il y a de l'autre côté aussi ? Au pire on fait les autres questions pour le moment.

11. Théo : Ouais parce que là si on n'y arrive pas... Alors... Tu lis la moitié je lis la moitié ?

12. Adrien : Ah ok Ou non on lit tous les deux dans sa tête.

13. Théo : Maitresse ça veut dire quoi en tôle?

14. Adrien : Où ça ?

15. Enseignante : En tôle? Ah c'est une matière, c'est comme les toits tu sais [montre le geste de l'ondulation des toits]. C'est pas très explicite comme ça. En fer comme ça là [dessine au tableau].

16. Théo : Ah oui !

17. Adrien : Les bateaux à moteur, enfin pas à moteur, à vapeur qui ont permis d'avancer plus vite pendant les traversées.

18. Théo : Les bateaux... On met les bateaux...

19. Adrien : ... nommés les stearmers

20. Théo : Ah mais attends non non non, les bateaux à vapeur nommés... comment ça se dit ? Steamers [Adrien épelle le mot]

21. Théo : J'arrive pas à le dire, je sais pas si c'est un mot anglais. Il a été construit en tôle, en fait tôle, elle m’a dit la maitresse que c'était...

22. Adrien : Des grosses briques qui sont comme ça là ?

23. Théo : Oui tu vois comme ça dans les maisons, elle a dessiné un dessin au tableau. Alors... Qu’a permis le développement des chemins de fer et de la navigation maritime? Tu comprends la question ? [Adrien relit le document]

24. Adrien : Des marchandises... Chemins de fer... En fait, là il y a écrit " qu'est ce qui a permis le développement des chemins de fer et de la navigation maritime ? " et là il y a écrit "Le chemin de fer et la navigation maritime multiplie les possibilités de déplacement des idées, des hommes et des marchandises ". Donc c'est peut-être grâce aux marchandises le développement des chemins de fer et de la navigation.

25. Théo : Ah oui parce que à partir de Paris jusqu’à... Oui, ils partaient d'un truc pour aller à Paris et pour euh... car...

26. Adrien : Du coup on écrit la marchandise.

27. Théo : Non on fait une phrase ! Elle a permis de... elle a permis de quoi ? Comment ça s'appelle?

28. Adrien : Elle a permis... de transporter des trucs...

29. Théo : De transporter de la marchandise.

30. Adrien : Les marchandises hein, parce qu'il y en a plusieurs.

31. Théo [en rigolant] : Oui. J'ai mis la marchandise alors que c'est les marchandises, avec un s.

32. Adrien : Oui, bah du coup... J'allais pas en mettre. Alors ensuite... La naissance de l'avion... Là il y a écrit place en rouge sur la frise la date de naissance de l'avion et en bleu la date de naissance de l'automobile.

33. Théo: On fait d'abord la naissance de l'avion.

34. Adrien : D'accord. Bah l'avion déjà il est là donc... [Lecture du document] La naissance de l'avion...

35. Théo : Faut que tu prennes ton rouge.

36. Adrien : En 1850 je sais plus quoi. Tu prends des feutres ? C'était en 1890.

37. Théo : En 1880 du coup c'est là.

38. Adrien : Mais non 1890.

39. Théo : Mais pourquoi t’as fait ça ?

40. Adrien : Parce qu'il fallait, elle a dit place en rouge sur la frise la date de naissance de l'avion.

41. Théo : Ah bah oui t’as colorié le numéro c’est pareil. Moi je fais comme ça.

42. Adrien: Mais c'est en 1890 !

43. Théo : Oui oui.

44. Adrien : Bah pourquoi tu mets 1880 ?

45. Théo : ... 10.

46. Adrien : Oui c'est $1880 \ldots$ hein ? Ah oui mince pardon mais c'est moi...

47. Théo : T'inquiètes pas moi j'ai fais pareil.

48. Adrien : Ensuite la date de naissance de l'automobile et de l'avion... [lecture du document]. L'automobile à essence nait en $1866 \ldots 1866 .$.

49. Théo : Non faut pas écrire en rouge!

50. Adrien : Ah oui en bleu. 
51. Théo : Ah ouf olalala on a eu chaud là ! En 1000 combien déjà ?

52. Adrien : C'est en 1866, donc on va faire un peu là tu vois?!

53. Théo : Montre

54. Adrien : Là on va le faire au milieu là

55. Théo : On est d'accord ?

56. Adrien : Au milieu à moitié

57. Théo : Ouais... Bon bah on passe à l'autre question ?

58. Adrien : On passe à celle là du coup... J'ai un peu de mal à comprendre quand même... Il nous reste du temps maitresse ?

59. Enseignante : Oui oui allez-y!

60. Adrien : On a le temps

61. Théo : Oui pour relire, tu relis dans ta tête ça.

62. Adrien : Attends mais c'est peut-être là les réponses! Quelles sont les conséquences du développement des chemins de fer sur la commercialisation du lait dans la capitale?

63. Théo : Bah on lit chacun ça dans sa tête et après..

64. Adrien : Au pire toi tu lis ça et moi je lis ce texte.

65. Théo : D'accord.

66. Adrien : Attends ten ten... Il y a écrit depuis 10 ans des changements notables se sont produits dans les modes d'approvisionnement du lait.

67. Théo : de la fabrication du lait?

68. Adrien : Oui ! Enfin de l'approvisionnement...

69. Théo : Bah je comprends pas trop là ! [Adrien lit le texte]

70. Théo : Attends je lis ma feuille... [Lit le texte] ça veut dire que les chemins de fer...

71. Adrien : ... c'est grâce au lait

72. Théo : Ouais... bon on va demander à la maitresse, parce que je comprends pas bien...

73. Adrien : Attends c'est juste qu'on lit mal

74. Théo : On est fous ou je sais pas... Attends je lève la main. [Adrien lit le texte]

75. Théo : Sur le haut ils parlent que les premiers trains ils étaient équipés d'une machine à vapeur et que ça faisait tourner les roues de la locomotive, ça allait plus vite...

76. Enseignante : Théo?

77. Théo : Bah en fait avec Adrien on a fait tout.

78. Adrien : et en fait...

79. Théo : Et celle là on comprend pas parce que euh...

80. Enseignante: Alors qu'est ce que ça a changé l'invention des trains à vapeur et des chemins de fer ? Ça change quoi ? Qu'est ce que ça a permis de changer?

81. Théo : Mais avec le lait on comprend pas...

82. Enseignante [montre le texte] : Là ça parle de lait, on vous parle du lait. L'approvisionnement en lait de Paris. C'est avec cet exemple.

83. Adrien : Ah ! le lait il va à Paris avec les trains ?

84. Enseignante : Je sais pas cherchez, allez y.

85. Théo : Bon d'accord on lit. Han j'ai pigé !

86. Adrien : T'as compris?

87. Théo : Ah j'ai pigé ! En fait c'est euh... c'est euh... c'est des gens qui achetaient aux fermiers du lait...

88. Adrien : .... et que du coup le lait...

89. Théo : ... le lait il allait dans les wagons pour aller jusqu’à.... jusqu’à la destination.

90. Adrien : Oui Paris!

91. Théo : Oui voilà

92. Adrien : Donc on met euh...

93. Théo : Attends on lit la question [lit la question]. Bah on va demander à la maitresse qu'est-ce qu'on peut mettre, on sait ça veut dire quoi mais on sait pas comment expliquer dans notre phrase réponse... Elle a servi aux chemins de fer ou je sais pas quoi... 94. Adrien : Bah au pire on peut mettre ce qu'on a dit

95. Théo : Attends non non!

96. Adrien : Les gens achètent le lait aux fermiers et donnent ensuite aux trains...

97. Théo : Maitresse ? Avec Adrien on sait ce que ça veut dire la... bah euh... que c'est des gens qui commandent aux fermiers des produits...

98. Enseignante : Ok

99. Théo: On n'arrive pas à le mettre sur la phrase

100. Enseignante : Alors c'est des gens qui commandent aux fermiers des produits d'accord ? Et donc comment on faisait avant ? Où est ce qu'ils allaient les produits ? Est-ce qu'ils allaient jusqu'à Paris ? Quand vous étiez à La Baule et que vous faisiez du lait ? 101. Adrien : Bah dans des usines!

102. Enseignante : ça n'existait pas. Vous habitez à La Baule et vous faites du lait, vous avez 10 vaches et vous faites du lait est ce que vous pouvez amener votre lait jusqu’à Paris ?

103. Théo: Bah non. On met dans un train qui va jusqu’à Paris.

104. Enseignante : Maintenant oui, mais avant on pouvait pas il n'y avait pas de trains.

105. Théo : De wagons?

106. Enseignante : Il n’y avait rien! Ça n’avançait pas vous alliez l'amener en calèche mais en calèche vous étiez pas arrivé au bout 
de vos peines hein... Le lait n'était plus bon avant qu'il arrive. Donc maintenant qu'est-ce que ça permet ? Vous m'avez dit on le met dans des trains pour l'amener jusqu’à Paris.

107. Théo : ça permet de transporter?

108. Enseignante : ça permet de transporter effectivement, ça permet de transporter sur... c'est comment la distance maintenant ? Avant vous donniez votre lait au village d'à côté mais vous ne donniez pas votre lait jusqu’à Paris si vous habitiez à La Baule ! 109. Théo : Non.

110. Enseignante : Donc maintenant c'est une plus grande distance, vous pouvez aller plus vite et plus loin, parce que le train ça va vite et ça va plus loin qu'avant.

111. Théo : Mais on n’arrive pas à former notre phrase...

112. Enseignante : Maintenant je vous ai donné tout vous avez plus qu’à...

113. Adrien : Aie aie aie...

114. Théo : Donc on fait... [relecture de la question] bah elle permet de transporter du lait à plus grande distance...

115. Adrien : Ah mais grave! Il permet de transporter du lait en plus grande distance. Terminé !

Mots-clés : Communauté discursive ; histoire ; enquête ; problématisation

\section{Bedingungen für den Wechsel der investigativen Sprachpraxis in der Schulgeschichte: eine Studie an einer Grundschule}

Zusammenfassung

Der Artikel befasst sich mit Fallstudien, die zeigen, dass Schüler/innen ein wissenschaftliches Vorgehen demonstrieren können, das denen von Wissenschaftlern ähnlich ist. Im Geschichtsunterricht sind zwei Arten von Vorgehensweisen relevant: dokumentarisch-kritische Verfahren zur Wahrheitsfindung oder die Analyse von Spuren der Vergangenheit verbunden mit der Suche nach deren Bedeutung. Wir dokumentieren die Bedingungen für den Wechsel von der einen zur anderen Vorgehensweise, indem wir dies anhand autonomer Arbeiten von Schüler/innen am Ende der Grundschule analysieren. Diese Analyse stellt die Vorstellung in Frage, dass die Entwicklung von Fähigkeiten durch die Beherrschung von Regeln, die historisches Denken beschreiben, veranlasst wird, und unterstreicht die Annahme, dass akademische Fähigkeiten Schüler/innen helfen können, über die schultypischen Gewohnheiten hinauszugehen.

Schlagworte: Diskursive Gemeinschaft; Geschichte; Untersuchung; Problematisierung

\section{Condizioni per la trasformazione delle pratiche linguistiche di indagine nella storia della scuola: un caso studio nella scuola elementare}

\section{Riassunto}

Larticolo esamina casi studio che mostrano le capacità degli studenti di mobilitare pratiche simili a quelle degli scienziati. Nello studio della storia sono possibili due tipi di indagini: seguire le procedure di critica documentaria per stabilire una verità, $o$ articolare la ricerca della verità analizzando le tracce del passato e cercando un significato. Documentiamo le condizioni del passaggio dall'una all'altra di questi tipi di indagine analizzando un caso di lavoro autonomo degli alunni alla fine della scuola primaria. Questo caso mette in discussione l'idea che lo sviluppo delle competenze abbia luogo attraverso la padronanza delle regole che descrivono il pensiero storico, e sottolinea il fatto che le competenze accademiche possono aiutare gli studenti ad andare oltre le abitudini della disciplina scolastica.

Parole chiave: Comunità discorsiva; storia; indagine; problematizzazione 


\section{Conditions for the Transformation of Investigative Language Practices in School History: An Elementary School Case Study}

Summary

The article addresses case studies that show students' abilities to mobilize practices that have to do with those of scientists. Two types of investigations are thus possible in history classes: following documentary criticism procedures to establish truth, or articulating the search for truth by analyzing traces of the past and searching for meaning. We document the conditions of transition from one type of investigation to the other by analyzing a case of autonomous work by pupils at the end of primary school. This case questions the idea that the development of skills would take place through the mastery of rules describing historical thinking, and it highlights the fact that academic skills can help students to go beyond the habits of school discipline.

Keywords: Discursive community; history; investigation; problematization

Sylvain Doussot, Docteur, habilité à diriger des recherche en didactique de l'histoire, Professeur des universités à l'Inspé de l'Université de Nantes, 4 chemin de Launay-Violette, F-44300 Nantes

E-Mail : sylvain.doussot@univ-nantes.fr 